\title{
Finger Vein Image Enhancement Based on Guided Tri-Gaussian Filters
}

\author{
Liping Zhang ${ }^{1,4} \oplus$, Xinran Wang ${ }^{2, *} \oplus$, Xiaoli Dong ${ }^{1,4, *} \oplus$, Linjun Sun ${ }^{1,4} \oplus$, Weiwei Cai ${ }^{3} \oplus$ \\ and Xin Ning ${ }^{1,4}$ \\ ${ }^{1}$ Cognitive Computing Technology Joint Laboratory, Wave Group, Beijing 10008, China \\ ${ }^{2}$ Beijing University of Posts and Telecommunications, Beijing 100876, China \\ ${ }^{3}$ Central South University of Forestry and Technology, Changsha 410004, China \\ ${ }^{4}$ Institute of Semiconductors, Chinese Academy of Sciences, Beijing 100083, China \\ * Corresponding author: Xinran Wang (wangxr@bupt.edu.cn) and Xiaoli Dong (dongxiaoli@semi.ac.cn)
}

Manuscript Revised 23 April 2021; Accepted 26 April 2021; Published 27 April 2021

Academic Editor: Hayley Dawson (D)

Abstract: In the process of image acquisition, the contrast between veins and non-veins in finger vein images is not high due to the influence of the fuzzy light source, skin scattering and finger movement. To solve this problem, a finger vein image enhancement method is proposed (GTGFs), which enhances finger vein patterns by setting guided image as input image firstly. On this basis, the tri-Gaussian model is based on disinhibitory properties of the concentric receptive field used to locally enhancing the image. The parameters of the tri-Gaussian model are determined based on the finger vein width information. The experiment results show that the proposed enhancement method can significantly enhance the finger vein patterns and improve the recognition effect of the methods based on vein pattern segmentation.

Index Terms: Finger vein image, Enhancement method, Guided filter, Tri-Gaussian filter.

\section{Introduction}

Finger vein recognition is a kind of biometric recognition technology $[1,2]$. The principle of this technology is to use the absorption property of hemoglobin in finger vein blood to short-wave near-infrared light to collect vein image and then carry out identity recognition. Thus, when near-infrared light $(760-850 \mathrm{~nm})$ is applied to the finger, dark pixels are formed in the vein region in the captured images. However, blurring light source, skin scattering, uneven finger thickness, various vascular thickness, and other factors will result in low contrast between vein pattern region and non-pattern region in finger vein image, which stands in the way of later extracting feature. Such kind of interference factor is severe for methods based on detail point or grain segmentation. Many previous studies have developed different finger vein image enhancement methods to overcome the problem of finger vein recognition performance degradation caused by fuzzy input image.

Among the extant methods of finger vein image enhancement, the most common one is based on various filters. Zhang et al. proposed Combination of Gray-Level Grouping and Circular Gabor Filter, an enhancement method, which can provide the contrast of vein images and reduce illumination changes, and Circular Gabor Filter is used to enhance vein patterns [3]. Yang et al. proposed a Multi-Channel Gabor Filter enhancement method, a reconstruction rule was used to mix the venous information obtained by Gabor filters with different scales and orientations [4]. Lee et al. proposed an enhancement method to deal with skin scattering and optical blurring problems. This method applies Point Diffusion Equation and Least Square Filter with Constraints [5]. Li et al. used Ridgelet Transform to enhance the high-frequency information of images, which could better deal with the singularity and edge 
enhancement of the two-dimensional curve of finger vein [6]. Vlachos et al. adopted a compound filter to enhance venous details [7]. Shin et al. proposed a fuzzy-based fusion enhancement method with Gabor and Retinex filtering[8]. Cao et al. proposed an enhancement method based on edge detection weighted guided filtering to solve the problem that the guided filtering could not highlight the detailed information of vein texture. In this method, an edge operator was set to punish the normalization factor in the guided filtering to improve the effect [9]. Ho et al. proposed an enhancement method based on using the direction and thickness of vein lines, which used adaptive Gabor to select an optimal direction of veins, applied morphological operations in the filtered image, and finally achieved the enhancement effect by fusion with the original image [10]. Shi et al. proposed an enhancement method based on scattering removal and even symmetric Gabor filter banks, which takes into account light propagation behavior in biological tissues and changes in the direction and diameter of vein patterns [11]. Pi et al. firstly used edgepreserving filter reducing noise and elliptical high-pass filter enhancing images, and on this basis, used histogram equalization to enhance image contrast [12]. Wang et al. designed a morphological image enhancement method by using genetic programming [13]. To solve the problem of light scattering more effectively, Yang et al. proposed a multi-layer PSF and BOM fusion enhancement method [14]. Although these methods can enhance finger vein patterns and take noise suppression into consideration, the noise will also be enhanced in the processed image, which also has a negative impact on recognition.

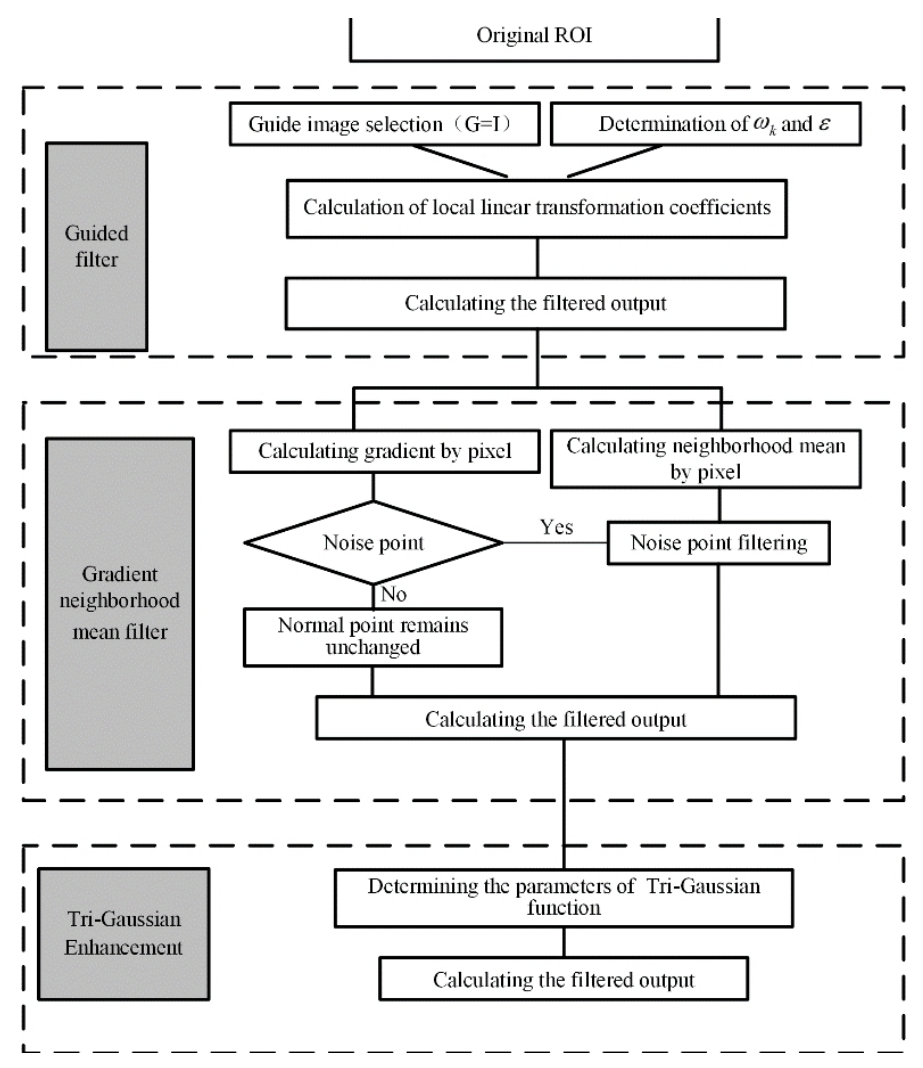

Fig.1. An overview of the GTGFs finger vein enhancement method.

\section{Methods}

A GTGFs finger vein image enhancement method is proposed in this paper. The method firstly enhanced the vein image through the guided filter, then designed a simple gradient neighborhood average filtering method to filter out noise caused by the equipment, and finally conducted a local enhancement by applying the tri-Gaussian model on the classic lateral suppression receptive field. Figure 1 shows the overall processing flow chart of the proposed method. The input is the ROI image through the finger vein, and the output is the final processing result. The size and resolution of the image are not changed during the whole processing process.

\subsection{Guided Filter}

The guided filter is essentially a linear model [15]. By constructing a general linear transform filtering process, which contains the guided graph, the input image, and the output image, where the boot diagram and the input image are all known, the value of the output image in pixel $i$ can be represented as a weighted average within a window.

$$
q_{i}=\sum_{j} W_{i j}(G) I_{j},
$$

where $i, j$ is the pixel index, weight $W_{i j}$ determined by guided graph $\mathrm{G}$, which is completely independent from the input image.

In the guided filtering, it is assumed that there is a local linear transformation relation between the guide graph $G$ and the filtering output $q$, that is, it is assumed that $q$ is the linear transformation of $G$ in a two-dimensional window $\omega_{k}$ centered on pixel $\mathrm{k}$, which is expressed as follows:

$$
q_{i}=a_{k} G_{i}+b_{k}, \forall i \in \omega_{k} .
$$

In the above formula, $\left(a_{k}, b_{k}\right)$ are the fixed linear coefficients in the window. Because of $\nabla q=a \nabla G$, the output image $q$ and $G$ have the same edge, in other words, guide graph $G$ can determine the weights used in the weighted average. The guide graph can be a single image or equal to the input image $p$. When the input image is used as the guide graph, the guided filtering is an edge-preserving filter.

To determine the parameters $\left(a_{k}, b_{k}\right)$, building a cost function that calculates the difference between input and output in each window $\omega_{k}$.

$$
\min E\left(a_{k}, b_{k}\right)=\min \sum_{i \in w_{k}}\left[\left(a_{k} G_{i}+b_{k}-I_{i}\right)^{2}+\varepsilon a_{k}^{2}\right],
$$

where $\varepsilon$ is a regularization parameter to prevent excessive size. The optimal solution of the above equation can be obtained by using linear regression method: 


$$
\left\{\begin{array}{c}
a_{k}=\frac{\frac{1}{|\omega|} \sum_{i \in w_{k}} G_{i} I_{i}-\mu_{k} \overline{I_{k}}}{\sigma_{k}^{2}+\varepsilon} \\
b_{k}=\bar{I}_{k}-a_{k} \mu_{k}
\end{array},\right.
$$

where, $\mu_{k}$ and $\sigma_{\mathrm{k}}^{2}$ are respectively the mean value and variance of the guide graph $G$ in the window, $|\omega|$ is the number of pixels in the window $\omega_{k}$, and $\bar{I}_{k}$ is the mean value of the input image $I$ in the window $\omega_{k}$. According to the obtained coefficients, the pixel value of the corresponding point of the output image can be calculated:

$$
q_{i}=\frac{1}{|\omega|} \sum_{k, i \in w_{k}}\left(a_{k} I_{i}+b_{k}\right)=\overline{a_{i}} I_{i}+\overline{b_{i}},
$$

where, $\bar{a}_{i}, \bar{b}_{i}$ is the mean value of $a_{k}, b_{k}$ of all windows that cover pixel point $i$.

Substitute the above results into Equation (1) to obtain the expression of the filter weight parameters:

$$
W_{i j}(G)=\frac{1}{|\omega|^{2}} \sum_{(i, j) \in \omega_{k}}\left(1+\frac{\left(G_{i}-\mu_{k}\right)\left(G_{j}-\mu_{k}\right)}{\sigma_{k}^{2}+\varepsilon}\right) .
$$

Because the information provided by the guide graph is mainly used to indicate which is the edge or which is a region, the local linear relationship between graph $G$ and $q$ is designed. If the guide graph detects that this area is detected, then it will smooth the filtering result. If the guided graph detects that the region is an edge, it will try to keep the edge information in the filtering results. When the guided graph $G$ and the input graph I are the same image, it becomes the self-guided filtering, which is used as an image enhancement filter, and its processing effect is similar to that of the bilateral filter, where the window radius is the smoothing radius, and the parameter is the smoothing parameter. The larger the value is, the more obvious, the smoother it will be.

In the finger vein recognition task, the input is the finger vein image, and the vein patterns in the image are the information we need (which can be regarded as the edge information), while most of the rest of the finger body is the regional information is not essential. Therefore, guided filtering can be used to achieve finger vein patterns enhancement. In this paper, self-guided image filtering is adopted, which makes the guide graph $G$ equaling $I$.

\subsection{Gradient Neighborhood Mean Filter}

To filter the noise points enhanced by guided filtering and keep the pixel value of the normal points unchanged, a simple gradient neighborhood mean filtering method is adopted. The specific processing process is as follows:

(1) For each pixel point, set a neighborhood window $\mathrm{w}$ of a specific size to calculate the neighborhood average value corresponding to this point.

(2) Calculate the gradient values in the four directions of the pixel.
(3) If one of the four gradient values is greater than the set threshold, the point will be judged as an abnormal point. The neighborhood average value will replace the grey value of the abnormal point in Step 1, and the remaining points will keep the original pixel unchanged.

\subsection{Local Enhancement of Finger Vein Image Based on Tri-Gaussian}

Based on the classical receptive field model, the Disinhibitory Properties of the Concentric Receptive Field (DRF) [16] added an excitatory edge region, which mainly played a role in disinhibition. Compared with the middle zone and the surrounding zone, the edge zone has weaker intensity and a more extensive effective range, as shown in Fig. 2. The triGaussian model based on DRF [17] adds a Gaussian function based on the classical Gaussian difference model to represent the action mechanism of the edge excitation region of DRF. The mathematical formula of the specific model is as follows:

$$
\begin{array}{r}
G(x, y)=A_{1} \exp \left(-\frac{x^{2}+y^{2}}{2 \sigma_{1}^{2}}\right)- \\
A_{2} \exp \left(-\frac{x^{2}+y^{2}}{2 \sigma_{2}^{2}}\right)+, \\
A_{3} \exp \left(-\frac{x^{2}+y^{2}}{2 \sigma_{3}^{2}}\right)
\end{array}
$$

where, the meaning of $A_{1}, A_{2}, \sigma_{1}, \sigma_{2}$ mean the same as in classical receptive field model, $A_{3}, \sigma_{3}$ are the peak coefficient and the standard deviation of Gaussian envelope of the edge region.

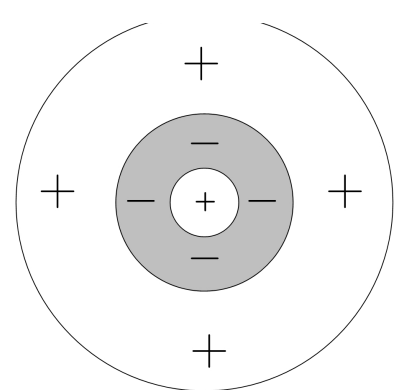

Fig.2. Disinhibitory model of the concentric receptive field.

The finger vein image is mainly composed of vein patterns and background information. The one that needs to be enhanced is vein patterns information. In addition to reflecting the network information such as the direction, crossing and connection of veins, finger vein image contains the information of vein width. For example, the main vein is wide, and the branch vein is narrow. Therefore, according to the structural properties of the finger vein image, by observing the ROI image of the SEMI-FV dataset (neither guided filtering nor gradient neighborhood means filtering changes the resolution and size of the image), it can be found that the vein width of the dataset occupies about 6-pixel width. In order to enhance the vein 
patterns, set $\sigma_{1}=\frac{t}{2}=3$. Combined with the response properties of the tri-Gaussian model [16], the value of 6 parameters in Equation (7) is finally determined, as shown in Table 1.
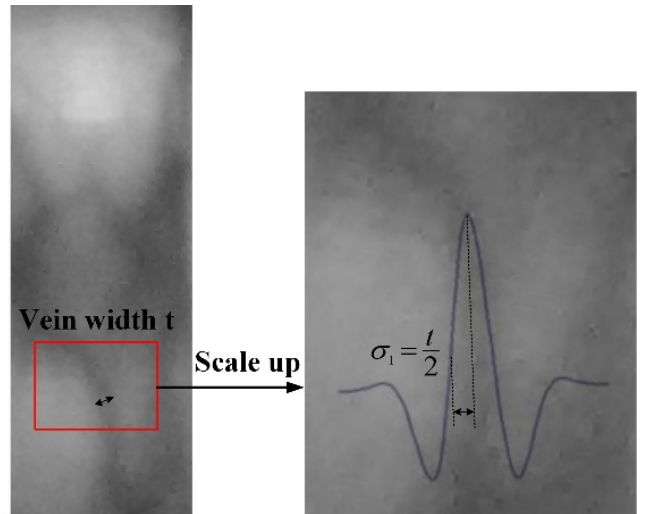

Fig.3. The sketch map of tri-Guassian model parameter setting.

Table 1. Parameter values of tri-Guassian model.

\begin{tabular}{|c|c|c|}
\hline Region & A & $\boldsymbol{\sigma}$ \\
\hline Central area & 14 & 3 \\
\hline Surrounding area & 2 & 7 \\
\hline Edge area & 1 & 11 \\
\hline
\end{tabular}

\section{Experimental Results}

The finger vein database for experiment was established by using the self-developed finger vein image acquisition device $[19,20]$. The self-built finger vein dataset contains 2,610 images from 29 people. Index finger, middle finger and ring finger are collected from each person's left and right hands. Each collection is carried out in a cycle of 6 fingers to increase the randomness of each finger press. The original collected image is shown in Fig. 4, with an image size of $640 \times 480$.
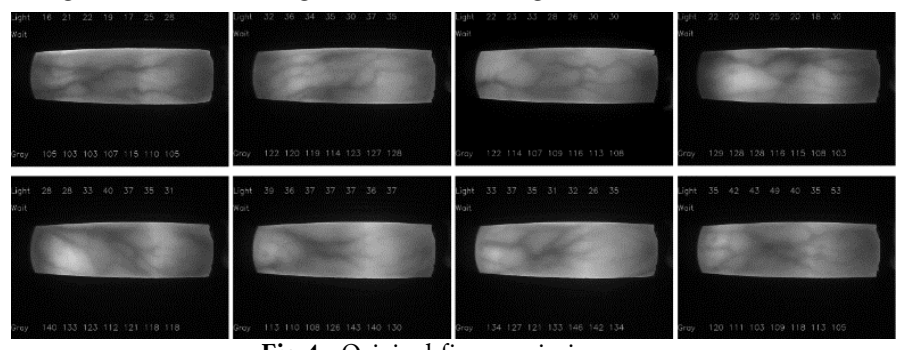

We perform two types of experiments to validate the proposed GTGFs. First, we analyze the visual effects of each part of the proposed method. Then, we binarize the output image after each part of the proposed method and test the recognize performance of the binary images. In identification test, each finger being treated as an independent object. With each object, the first five images are regarded as the training images, the remaining 10 images used in the test process. By calculating the compatibility between the two images and using the nearest neighbor classification, we obtain the measurement results of each test image for each category. Taking the Equal Error Rate (EER) as the measurement index, there were 1740 positive sample matching times $(174 \times 10)$ and 301,020 negative sample matching times $(174 \times 173 \times 10)$.

\subsection{Visual Effect After Processing}

As shown in Fig.5, each image shows the effect after processing of each step in the flow. Based on vein enhancement with guided filter, vein patterns enhanced after processing. However, noise can also be amplified. Through carefully observing the enhanced image noise points, we found that the part of the noise may be caused by acquisition equipment (the dust on the acquisition camera), and its noise type is similar to Gaussian noise. Although the principle of the designed gradient neighborhood mean filtering is simple, it can effectively filter the noise of the image without changing the effective point information of the image and keep the original vein patterns information of the image as much as possible. Although the normal pixel value of the image can not be changed by the gradient neighborhood mean filtering, the image near the abnormal point is obviously blurred after filtering. On this basis, adding the tri-Gaussian image enhancement algorithm process can further enhance the vein patterns, especially in the local area.

\subsection{Binarization After Processing}

The pre-processed image is binarized by setting a fixed threshold value, and then Hausdorff distance matching is carried out directly on the binarized image matching. The binary images processed by each method are shown in Fig. 6, and the EER results of different pre-processing methods are shown in Table 2.

Table 2. Comparison of EER values on binary image using Hausdorff distance match after different pre-processing on binary images.

\begin{tabular}{|c|c|}
\hline Methods & EER/\% \\
\hline Original ROI & 14.33 \\
\hline Guided Filter & 12.36 \\
\hline $\begin{array}{c}\text { Guided Filter + } \\
\text { Gradient Neighborhood Mean Filter }\end{array}$ & 11.24 \\
\hline GTGFs & 10.78 \\
\hline
\end{tabular}

In the above two groups of experiments, it can be seen from the results of Experiment 1 that after the processing of the proposed method, vein images have a significant enhancement effect, and the noise brought by the equipment can be filtered, and the visual effect is noticeable. Experiment 2 shows that the proposed finger vein image enhancement method can improve the accuracy of vein segmentation and recognition. However, since the binarization method in Experiment 2 adopts the simplest threshold binarization, and the matching is also simple Hausdorff distance matching, the recognition effect is poor. 


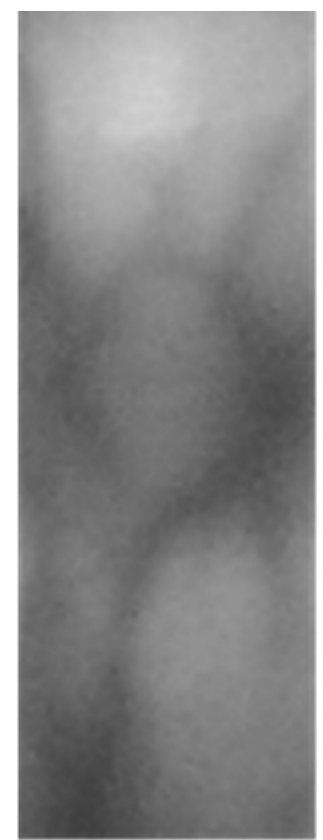

(a) Original image

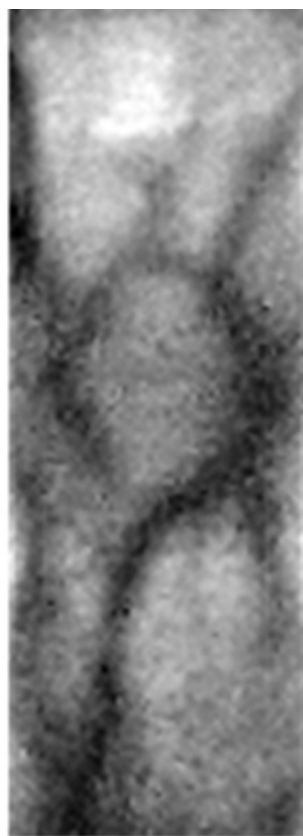

(b) Image after guided filtering

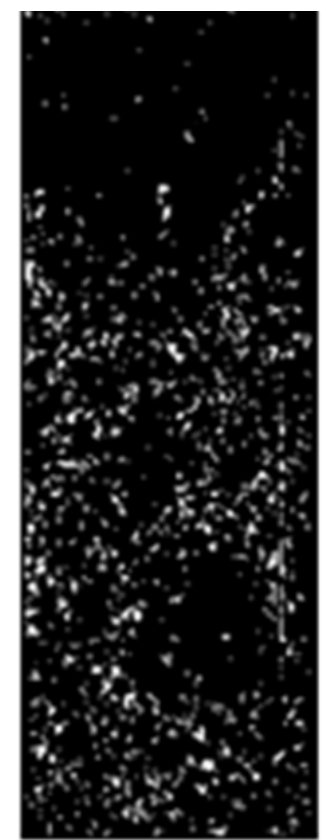

(c) Noise points filtered by gradient neighborhood mean filter

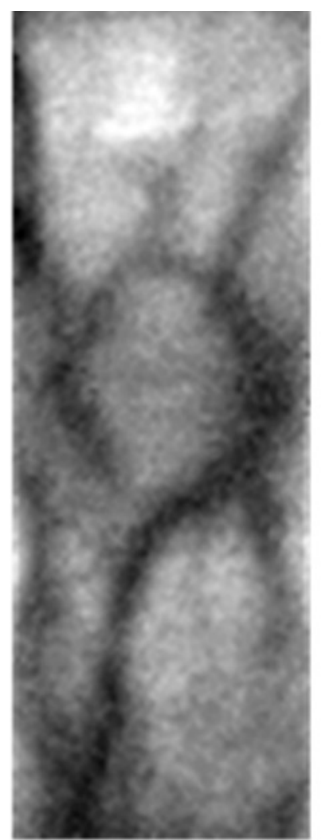

(d) Image after gradient neighborhood mean filtering

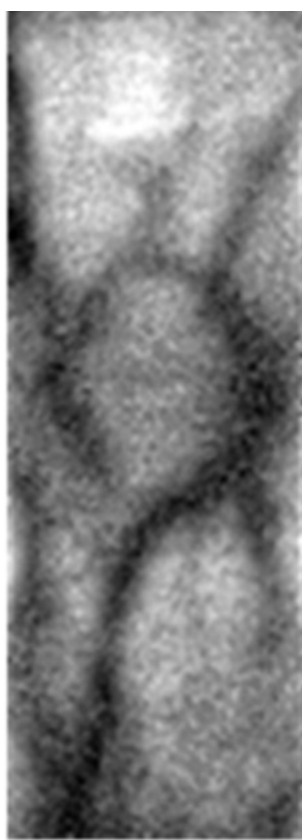

(e) Image after GTGFs

Fig.5. The enhancement performance comparison in each stage of GTGFs.

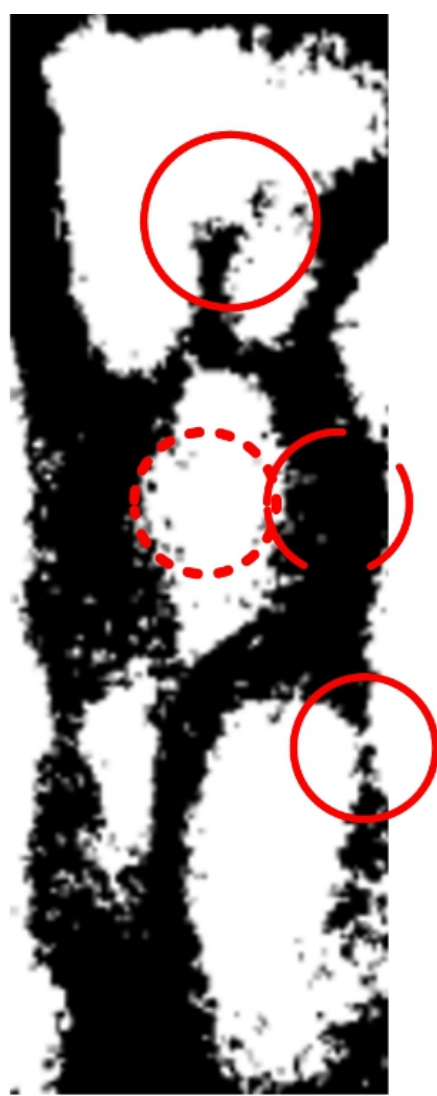

(a) Binarization of original image

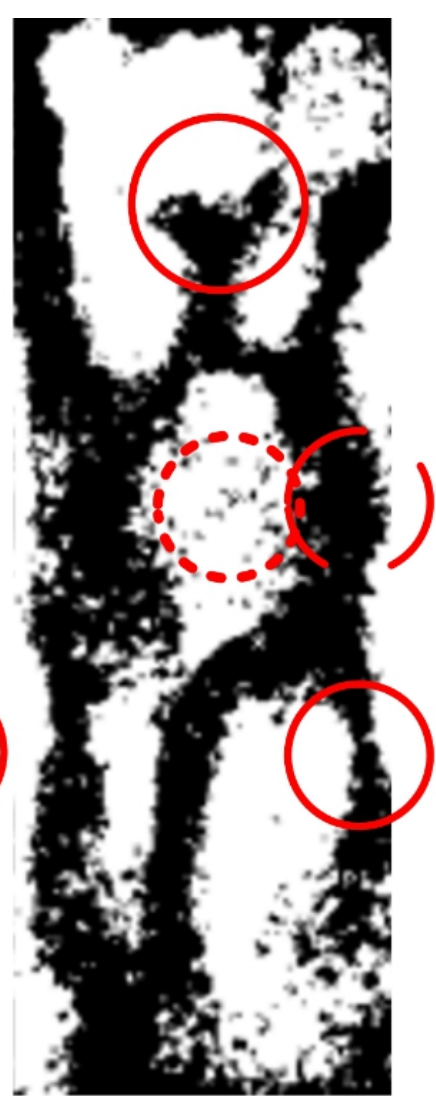

(b) Binarization of the image after guided filtering

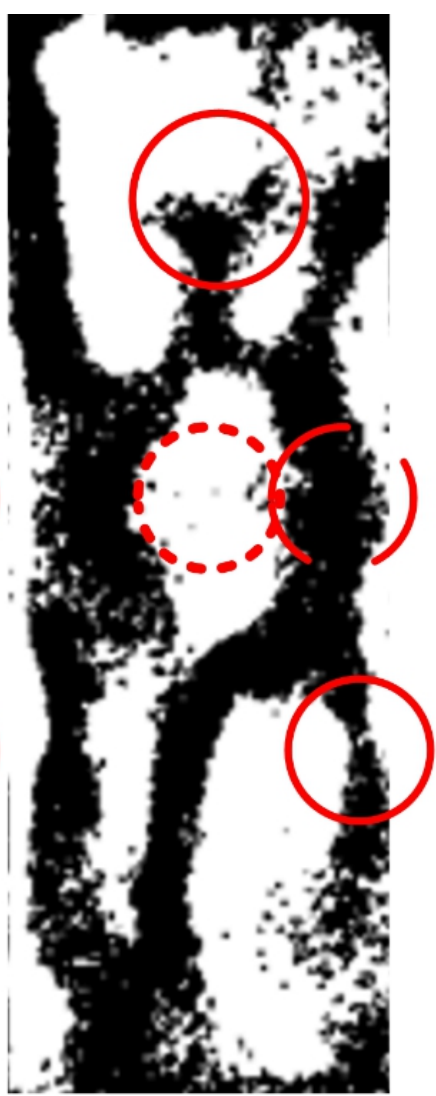

(c) Binarization of the image after gradient neighborhood mean filtering

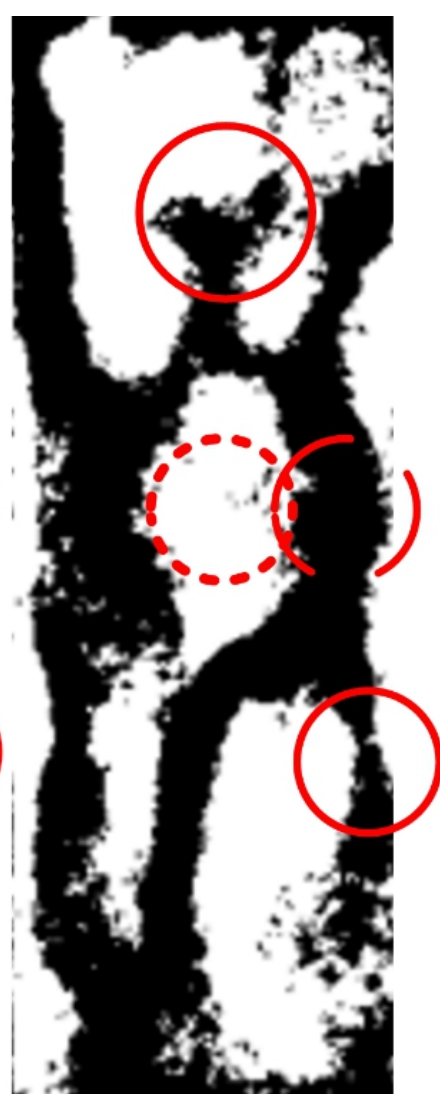

(d) Binarization of the image after Tri-Gaussian filtering
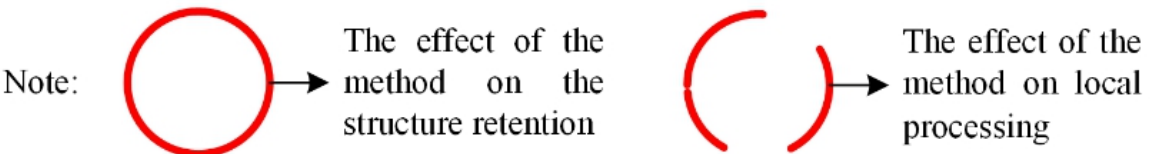

Fig.6. Binary images after different pre-processing.

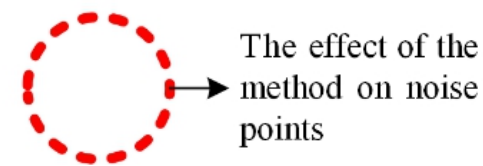




\section{Conclusion}

In this paper, an image enhancement method of finger vein combining guided filtering and tri-Gaussian model is proposed. The method contains three parts: guided filtering, gradient neighborhood mean filtering and three-Gaussian image enhancement. First, a self-guided filter is formed by setting the input image as the guide graph to enhance the finger vein patterns. In order to filter out the noise points which are also enhanced, a simple gradient neighborhood mean filtering strategy is adopted. Finally, according to the properties of finger vein patterns, parameters were set and the image of finger vein was enhanced by the tri-Gaussian model. The experimental results show that the image processed by the method can achieve good results in the aspects of preserving the structure of finger vein, local processing and suppressing noise points.

\section{Acknowledgement}

This work was supported by Shenzhen Wave Kingdom Co., Ltd.

\section{References}

[1] Zhang, Y., Li, W., Zhang, L., Ning, X., Sun, L., \& Lu, Y. (2019). Adaptive learning Gabor filter for finger-vein recognition. IEEE Access, 7, 159821-159830.

[2] Ning, X., Li, W., Tang, B., \& He, H. (2018). BULDP: biomimetic uncorrelated locality discriminant projection for feature extraction in face recognition. IEEE Transactions on Image Processing, 27(5), 2575-2586.

[3] Zhang, J., \& Yang, J. (2009, December). Finger-vein image enhancement based on combination of gray-level grouping and circular gabor filter. In 2009 International Conference on Information Engineering and Computer Science (pp. 1-4).

[4] Yang, J., \& Yang, J. (2009, September). Multi-channel gabor filter design for finger-vein image enhancement. In 2009 Fifth International Conference on Image and Graphics (pp. 87-91).

[5] Lee, E. C., \& Park, K. R. (2011). Image restoration of skin scattering and optical blurring for finger vein recognition. Optics and Lasers in Engineering, 49(7), 816-828.

[6] Li, H. B., Yu, C. B., Zhang, D. M., \& Zhou, Z. M. (2010, September). The Study on Finger Vein Image Enhancement Based on Ridgelet Transformation. In 2010 6th International Conference on Wireless Communications Networking and Mobile Computing (WiCOM) (pp. 1-4).

[7] Vlachos, M., \& Dermatas, E. (2008, May). Vein segmentation in infrared images using compound enhancing and crisp clustering. In International Conference on Computer Vision Systems (pp. 393402). Springer, Berlin, Heidelberg.

[8] Shin, K. Y., Park, Y. H., Nguyen, D. T., \& Park, K. R. (2014). Finger-vein image enhancement using a fuzzy-based fusion method with gabor and retinex filtering. Sensors, 14(2), 30953129.

[9] Wei, C., Huabin, W., Jun, S., Rui, Y., \& Liang, T. (2017). Enhancement algorithm of finger vein image based on weighted guided filter with edge detection. Laser \& Optoelectronics Progress, $54(2), 021007$.
[10] Park, Y. H., \& Park, K. R. (2012). Image quality enhancement using the direction and thickness of vein lines for finger-vein recognition. International Journal of Advanced Robotic Systems, 9(4), 154.

[11] Shi, Y., \& Yang, J. (2012, October). Image restoration and enhancement for finger-vein recognition. In 2012 IEEE 11th International Conference on Signal Processing (Vol. 3, pp. 16051608).

[12] Pi, W., Shin, J., \& Park, D. (2010, August). An effective quality improvement approach for low quality finger vein image. In 2010 International Conference on Electronics and Information Engineering (Vol. 1, pp. V1-424).

[13] Wang, J., \& Tan, Y. (2011, July). Morphological image enhancement procedure design by using genetic programming. In Proceedings of the 13th annual conference on Genetic and evolutionary computation (pp. 1435-1442).

[14] Yang, J., Zhang, B., \& Shi, Y. (2012). Scattering removal for finger-vein image restoration. Sensors, $12(3), 3627-3640$.

[15] He, K., Sun, J., \& Tang, X. (2012). Guided image filtering. IEEE transactions on pattern analysis and machine intelligence, 35(6), 1397-1409.

[16] Chao-Yi, L., Xing, P., \& Yi-Xiong, Z. (1991). Role of the extensive area outside the $\mathrm{X}$-cell receptive field in brightness information transmission. Vision Research, 31(9), 1529-1540.

[17] Chao-Yi, L., Yi-Xiong, Z., Xing, P., Fang-Tu, Q., Cheng-Quan, T., \& Xing-Zhen, X. (1992). Extensive disinhibitory region beyond the classical receptive field of cat retinal ganglion cells. Vision Research, 32(2), 219-228.

[18] Ning, X., Li, W., \& Liu, W. (2017). A fast single image haze removal method based on human retina property. IEICE TRANSACTIONS on Information and Systems, 100(1), 211-214.

[19] Zhang, L., Li, W. J., Ning, X., Dong, X. L., \& Liu, W. J. (2018). A Finger Vein Recognition Method Based on Histogram of Oriented Lines and (2D) 2FPCA [J]. Journal of Computer-Aided Design es Computer Graphics, 30(2), 254-261.

[20] Zhang, L., Li, W., \& Ning, X. (2020). A Local Descriptor with Physiological Characteristic for Finger Vein Recognition. arXiv preprint arXiv:2004.07489.

\section{Biographies}

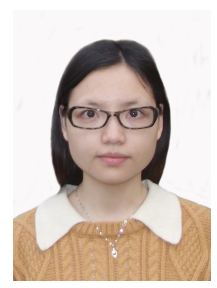

Liping Zhang received her Ph.D. from Institute of Semiconductors, Chinese Academy of Sciences in 2018. where she is currently an assistant research fellow. Her research interests include biometrics, pattern analysis. She is a member of IEEE.

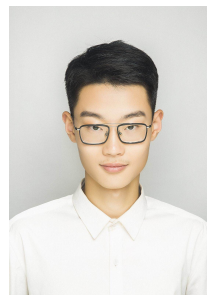

Xinran Wang, an undergraduate student at Beijing University of Posts and Telecommunications, whose research interests include machine learning, deep learning, collaborative training, and image generation algorithms. 


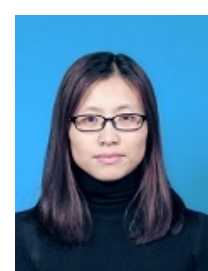

Xiaoli Dong received her Ph.D. from Institute of Semiconductors, Chinese Academy of Sciences in 2018. where she is currently an assistant research fellow. Her research interests include image processing, pattern recognition. She is a member of IEEE.

Linjun Sun received the Ph.D. degree in electronic circuit and system from the Institute of Semiconductors, Chinese Academy of Sciences, in 2020. He is currently a Post-Doctoral Research Fellow with the Laboratory of Artificial Neural Networks and High Speed Circuits, Institute of Semiconductors, Chinese Academy of Sciences. His main research interests are computer vision and deep learning. He is a student member of IEEE.

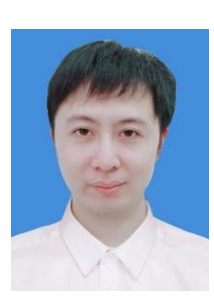

Weiwei Cai is currently practicing at the Laboratory of Artificial Neural Networks and High Speed Circuits, Institute of Semiconductors, Chinese Academy of Sciences, Prior to that, he worked with IT industry for more than ten years in the roles of an System Architect and the Program Manager. His research interests include machine learning, deep learning, and computer vision.

Xin Ning received the B.S. degree in software engineering from Xinjiang University, Wulumuqi, China, in 2012, and the Ph.D. degree in electronic circuit and system from the Institute of Semiconductors, Chinese Academy of Sciences, in 2017. He is currently an Associate Professor with the Laboratory of Artificial Neural Networks and High Speed Circuits, Institute of Semiconductors, Chinese Academy of Sciences. He has authored or co-authored more than 40 papers in journals and refereed conferences. His current research interests include pattern recognition, computer vision, and image processing. 